\title{
Letter to editor: CoVID-19 pandemic and sleep disorders-a web survey in Italy
}

\author{
Pierluigi Innocenti ${ }^{1} \cdot$ Antonella Puzella $^{1} \cdot$ Maria Paola Mogavero ${ }^{2} \cdot$ Oliviero Bruni $^{3} \cdot$ Raffaele Ferri $^{4}$ (I)
}

Received: 14 May 2020 / Accepted: 20 June 2020 / Published online: 30 June 2020

(C) Fondazione Società Italiana di Neurologia 2020

\section{Dear Editor in Chief,}

The CoVID-19 pandemic has radically changed our daily lives; changing the rhythms of the day has provided reasons for anxiety, stress, and mood alterations that have affected our well-being and sleep. Yet a good sleep is even more important in this period because it increases our immune defenses [1] that help us fight the virus; it is fundamental for our mental balance but also for our physical well-being. Assirem ETS (Italian Association for Research and Education in Sleep Medicine) has launched a web-based survey to assess in a simple way the changes in sleep patterns associated with the lifestyle modifications occurred with the onset of the CoVID19 pandemic. For this reason, a questionnaire was arranged which included few preliminary questions about the subject entering it (such as sex, age range, and information about the eventual infection by CoVID-19), followed by an ad-hoc Italian adaptation of the Pittsburg Sleep Quality Index (PSQI) items [2, 3]. The questionnaire was arranged in order to collect the same information from each participant with reference to two months (the original PSQI investigates a series of sleep features in a time frame of one month): the month "preceding the onset of the CoVID-19 pandemic" and the following month. No precise dates were indicated because the pandemic started at different times in different geographic areas (even within the same country, as in Italy) and, thus, leaving this to the subjective perception of the emergency

Raffaele Ferri

rferri@oasi.en.it

1 Assirem ETS, Associazione Italiana per la Ricerca e l'Educazione nella Medicina del Sonno, Rome, Italy

2 Istituti Clinici Scientifici Maugeri, IRCCS, Scientific Institute of Pavia, Pavia, Italy

3 Department of Developmental and Social Psychology, Sapienza University, Rome, Italy

4 Oasi Research Institute, IRCCS, Troina, Italy period of each participant. The participation was voluntary, anonymous, and free; it was offered through the main social media, as well as through a link on the Assirem ETS web page. The survey was not supported by any grant or external funding.

Although this type of survey suffers from many methodological problems that are incompatible with a strictly scientific study protocol, it can nevertheless provide important indications on widespread phenomena in the population, in a short time, allowing to get important information in a timely and effective way, by also recruiting a large number of participants. In 10 days, 1035 complete questionnaires were collected and data were pooled into a database for further analysis.

Unexpectedly, $82.9 \%$ of the entire group were women and age was $\geq 18$ years ( $46.6 \%$ in the $30-50$ years range). Regarding education, $54 \%$ had a university degree or above and $40.7 \%$ completed high schools. Only $8(0.8 \%)$ reported having been infected by CoVID-19.

Even if the total number of hours of sleep at night was reported to remain substantially unchanged or only slightly reduced, bedtime and awakening times changed and were delayed by approximately $1-2 \mathrm{~h}$ and, even more significantly, the perceived quality of sleep changed, judged by more than half of the interviewees "quite or very bad" during the month following the pandemic onset ( $18 \%$ vs. $52.2 \%$; chi-square = 254, $p<0.00001)$.

Participants reported to go to bed $1-2 \mathrm{~h}$ later than before the CoVID-19 pandemic and to take longer to fall asleep (those taking $>1 \mathrm{~h}$ to fall asleep increased from 2.8 to $16 \%$; chisquare $=264, p<0.00001)$. Also the awakening was reported to be delayed by $1-2 \mathrm{~h}$, thus leaving the total sleep time unchanged, but with a tendency to decrease. Nighttime awakenings also were reported to be increased ( 3 out of 4 people woke up early at least once a week vs. 4 out of 10 people before the CoVID-19 pandemic; chi-square $=220$, $p<0.00001)$. People complaining of bad dreams increased from 1 out of 10 , before the pandemic, to 4 out of 10 after (chi-square $=273, p<0.00001)$. Although less noticeable, the 
CoVID-19 pandemic was associated to a $6 \%$ increase in number of people who took sleeping medications 3 or more times a week, while those who did not take them decreased by about $10 \%$ (chi-square $=40.5, p<0.0001$ ).

This survey, even if not supported by a rigorous and scientifically sound data collection, seems to provide interesting and important insights, highlighting a worsening trend of sleep patterns that needs to be monitored and followed carefully in order to prevent possible long-term consequences on people's health and the chronic development of some of the disorders associated to the sleep changes reported by the participants. Although total sleep duration was reported to be only slightly reduced, the responses to this survey indicate an increase of disordered sleep associated with the CoVID19 pandemic and characterized by a sleep phase delay (1-2 h), increase in nighttime or early awakenings, perception of decreased sleep quality, increased bad dreams, and increased use of sleeping pills. All these changes indicate an increased presence of symptoms of insomnia and nightmares which are considered to be the hallmarks of posttraumatic stress disorder [4] and can easily be interpreted as an obvious consequence of the psychological stress induced by the pandemic and the associated changes in lifestyle.

We believe that the disturbed sleep during Covid-19, especially in predisposed subjects, might represent a risk factor for the development of chronic insomnia or other sleep disorders. Great attention should be made and adequate countermeasures undertaken in order to prevent such a risk. These countermeasures should start from an intense awareness campaign to be conducted during the pandemic by health authorities, accompanied by education to sleep hygiene and, subsequently, by cognitive behavior therapy (an effective treatment that can also be carried out with web-based tools [5]) in affected subjects.

\section{Compliance with ethical standards}

Conflict of interest The authors declare that they have no conflict of interest.

Research involving human participants and/or animals Not Applicable.

Informed consent Not Applicable.

Ethical approval None.

\section{References}

1. Asif N, Iqbal R, Nazir CF (2017) Human immune system during sleep. Am J Clin Exp Immunol 6(6):92-96

2. Buysse DJ, Reynolds CF III, Monk TH, Berman SR, Kupfer DJ (1989) The Pittsburgh sleep quality index: a new instrument for psychiatric practice and research. Psychiatry Res 28(2):193-213

3. Curcio G, Tempesta D, Scarlata S, Marzano C, Moroni F, Rossini PM, Ferrara M, De Gennaro L (2013) Validity of the Italian version of the Pittsburgh Sleep Quality Index (PSQI). Neurol Sci 34(4):511519. https://doi.org/10.1007/s10072-012-1085-y

4. Weber FC, Norra C, Wetter TC (2020) Sleep disturbances and suicidality in posttraumatic stress disorder: an overview of the literature. Front Psych 11:167. https://doi.org/10.3389/fpsyt.2020.00167

5. Grierson AB, Hobbs MJ, Mason EC (2020) Self-guided online cognitive behavioural therapy for insomnia: a naturalistic evaluation in patients with potential psychiatric comorbidities. J Affect Disord 266:305-310. https://doi.org/10.1016/j.jad.2020.01.143

Publisher's note Springer Nature remains neutral with regard to jurisdictional claims in published maps and institutional affiliations. 